\title{
Acknowledgements-Part V, Chapter 12 Case studies
}

We would like to acknowledge the people who have been very helpful in illustrating the book with their written personal, concrete and professional experience in regards to virtual teams for our book's Part V Case studies. We warmly thank them for their contribution, time, application and enthusiasm.

Pierre Rosius, human resources director at Thomson Reuters, kindly agreed to share his experience of virtual teams. Thomson Reuters has implemented a new strategy and a new operating model in their Sales function. Pierre Rosius analyses the evolution of that organization and the impact of virtual teaming in an international context from a human resources point of view. He highlights the virtual teaming challenges and the consequences in Thomson Reuter's process.

Kim Poldner experienced an enthusiastic approach for virtual teaming through her online engagement in Eco Fashion World and a small dispersed team. Cultural differences, the difficulties to be 'all connected' whilst sharing a common goal at a distance, computer-mediated communication and the requisite team cohesion levels are some of the real barriers faced by Kim. She underlines the main considerations faced in a small virtual team, which is very instructive in comparison to larger international company structures.

Frederic Reiser realized as a virtual team member that virtual teaming requires adapted processes, especially when virtual teams are implemented together with organizational restructuring. Working process, new structure and adequate behaviours confronted with cultural diversity may foster unexpected results and may endanger the success of a strategy. Therefore, effective preparation for virtual teaming should not be underestimated.

Chloé Guerin Gosselin, a student in teaching history and geography, has chosen an e-learning programme offered by the University of Quebec at Trois-Rivières. Based on her experience, Chloé presents the advantages and challenges of virtual classrooms, virtual teamwork and online courses. She emphasizes that e-learning requires adapted behaviours and pedagogy 
techniques; in order to ensure effective and optimal teaching and learning outcomes.

Professor Silvester Ivanaj and Claire Bozon 
Silvester Ivanaj and Claire Bozon - 9781785369278

Downloaded from PubFactory at 04/26/2023 03:21:55PM 